\section{Beurteilung von Analysenergebnissen}

C. Vidal ${ }^{1}$ und W.-R. Külpmann ${ }^{2}$

${ }^{1}$ Landeskriminalamt Niedersachsen, Dezernat 53 „Chemie“, Hannover, Deutschland

${ }^{2}$ Hannover, Deutschland

Englischer Begriff evaluation of measurements

Beschreibung Messresultate müssen verschiedene Kriterien erfüllen, bevor sie als analytisch korrekt bewertet werden können. Die Kriterien umfassen:

- Qualitätskontrolle (Präzision, Richtigkeit) der zugehörigen Analysenserie

- Ausschluss der Störung durch analytische Einflussfaktoren
- Plausibilitätskontrolle (Delta-Check, Konstellations-, Extremwertkontrolle)

Es folgen der Vergleich mit dem zugehörigen Referenzintervall, dem therapeutischen Bereich oder mit der Entscheidungsgrenze (Transversalbeurteilung) sowie der Vergleich mit Vorwerten des Patienten (Longitudinalbeurteilung). Mit Abschluss der Vorgänge ist aus dem Analyseergebnis ein klinisch-chemischer Befund geworden.

\section{Literatur}

Stamm D, Büttner J (1995) Beurteilung klinisch-chemischer Analysenergebnisse. In: Greiling H, Gressner AM (Hrsg) Lehrbuch der klinischen Chemie und Pathobiochemie, 3. Aufl. Schattauer Verlag, Stuttgart, S 72-95 